\title{
Research on Green Innovation Incentive of Environmental Regulation
}

\author{
Hongfang Cai
}

\begin{abstract}
Gansu Forestry Polytechnic
\end{abstract}
741020

\begin{abstract}
:
Nowadays, China is facing increasingly severe social situation, for example,tighter resource constraints, serious environmental pollution, ecological environment degradation and so on. The demands for ecological transformation are increasing all over the world. At present, global environmental issues are more prominent, such as severe climate change and financial crisis, which provide certain development opportunities to promote the development of traditional economy to green economy. In order to ensure the true realization of green growth, it is necessary to speed up the adjustment of industrial structure and innovative breakthrough technology. In recent years, the development theme of our country is the construction of ecology and innovative technology. Since the 18th National Congress of the Communist Party of China and the Third Plenary Session of the 18th Central Committee of the Communist Party of China, the strategic position of the construction of ecological civilization and innovation driven development has been gradually defined. So, in order to in-depth implementation of the basic requirements of the scientific outlook on development, it is necessary to effectively link environmental regulations, in the field of technological innovation and environmental protection , We will find an effective mechanism between interaction and mutual benefit, so as to create good conditions for the realization of diversified and sustainable development, namely, economy, society and ecology.

Keywords: environmental regulation, green innovation, incentive research
\end{abstract}

\section{环境规制的绿色创新激励研究}

\section{蔡宏芳}

\section{甘肃林业职业技术学院 741020}

\section{摘要:}

现如今，我国面临着日益严峻的社会形势，即资源约束趋紧、环境污染严重、生态环境日益退化等， 对于生态化转型的诉求在全世界范围内都日益高涨。当下, 全球环境议题比较突出, 如严峻的气候变 化与金融危机等, 由此提供一定的发展机遇来促使传统经济向绿色经济发展, 而为了确保绿色增长的 真正实现，就需要加快调整产业结构与创新突破性技术。近年来，我国的发展主题在于建设生态与创 新技术，尤其是在党的十八大与十八届三中全会召开以来，建设生态文明与创新驱动发展的战略地位 逐渐明确下来, 基于此, 为实现对科学发展观基本要求的深入贯彻, 就需要有效连结环境规制, 在技 术创新、环境保护之间找到一种联动互利的有效机制，从而为实现多元化可持续发展创造良好条件， 即经济、社会与生态。

关键词: 环境规制; 绿色创新; 激励研究

\section{1. 前言}

以绿色创新导向的环境机制, 能够提供各种与之相 关的政策法规几何, 从而能够为企业、全社会绿色创新 活动的开展创造良好条件, 为促进技术创新与成果转化
水平的提高、绿色创新在制度环境方面不确定性的减少, 能够应在良好的创新环境 ${ }^{[1]}$ 。而成本抵偿效应与波特假 说的成立能够起到直接决定性作用, 且与绿色创新的强 弱激励过程相对应, 进而可以将两类干预政策类型衍生 出来。不同环境规制工具不存在优劣之分, 关键在于亟 需推动的技术类型与所处的实施背景, 如果多种工具能 
要性。波特假说由波特教授在 1991 年提出, 其能够站

够相辅相成, 则有助于将环境规制的绿色创新效应体现 出来。

\section{2. 绿色创新与环境规制}

\section{1 绿色创新}

所谓绿色创新主要是为了实现环境友好、可持续发 展而提供的各种创新活动, 其具有双重涵义, 即一般创 新范式、生态-经济-社会和谐共生, 其代表为技术创新, 基于此有助于实现人与人、自然、社会的和谐发展。其 中技术创新有广义与狭义之分, 狭义的在整体创新过程 中重视技术概念的重要性, 如关于生产技术、工艺、产 品或服务的研发和应用, 广义的不仅包括狭义层面的内 容, 还包括多种创新的结合, 如经营管理模式、生产组 织方式、机制体制以及市场营销方式等, 因此这种创新 活动比较复杂, 且影响深远。

关于绿色创新, 学术界存在不同看大, 许多学者能 够站在技术创新的角度, 实现对先进环境、绿色技术创 新等作用与影响的研究, 但是对于某一中心事物而言, 基于不同中心事物, 环境状态也有明显不同, 如主观人 文环境（自然或社会环境、制度环境等）。其基本特性 在于经济社会的可持续发展、经济效益和环境效果的协 调统一、创新主体的多元化等 ${ }^{[2]}$ 。

\section{2 环境规制}

规制主要是政府管理部门所采取的某种干预、限制 或约束行为, 旨在实现对市场机制内在问题的有效校正 与改善, 其基本出发点在于实现对公共权益的保护, 主 体在于政府。基于不同规制作用对象，可以包括三类， 即直接经济干预、生产者决策约束、消费者行为约束。 按照规制概念, 环境规制主要是针对市场活动所采取的 各种干预、约束行为, 旨在实现对污染的有效预防与控 制、生态环境的保护, 时政府开展环境管理的重要方式。 传统规制理论, 市场资源的配置效率深受环境外部性存 在的影响, 而环境规制有助于这一市场失灵情况的有效 纠正, 且环境规制是社会性规制的一种, 对于普通公众 所产生的社会收益相当广泛, 给污染者所带来的额外成 本相对集中。

\section{3. 环境规制对于绿色创新的激励机制}

\section{1 波特假说}

针对环境保护和经济增长, 传统经济学将其视为隐 含抵消关系的一种, 即在严格环境规制下有助于社会效 益的产生, 也会导致企业内部成本的增加, 从而不利于 提高市场竞争力, 且还会对所在国的国际竞争力产生重
在全新角度对环境规制、技术创新、市场竞争力之间的 作用关系进行有效的解释。其主要观点在于: 环境保护 与竞争力提升有助于共赢的实现、环境政策基于创新与 效率改进的动态影响、推动企业绿色创新的主体在于政 府、适当的政策环境对于绿色创新能够发挥强有力的推 动作用 ${ }^{[3]}$ 。

\section{2 环境规制作用下的绿色创新激励}

\subsection{1 环境规制的创新激励模型}

(1) 无环境规制时

假设在一个完全封闭的竞争市场中, 同质生产者有 $\mathrm{N}$ 非，最开始全部生产者使用的技术是一样的，且环境 外部性、规模生产外溢性同时存在于市场中，在各个时 期每个生产者所拥有的单位劳动为 1 , 且所有劳动均用 于某一消费品 $\mathrm{c}$ 的生产, 而劳动总功能给 $\mathrm{L}=\mathrm{Nl}^{[4]}$ 。

（2）引入环境规制

假设新技术 $\mathrm{g}$ 同样能够生产消费品 $\mathrm{c}$, 则劳动总供 给 $\mathrm{L} 1$ 在每个时期都为两种技术的劳动总和。

\subsection{2 环境规制的弱绿色创新激励}

按照波特假说, 为实现对企业负面环境行为的有效 约束, 政府会在环境标准、税费等外部环境成本内部化 的帮助下，鼓励企业积极的创新技术、改进效率，这对 于新增成本所造成的利润降低问题可以得到有效弥补, 从而有助于一定市场竞争力的形成。但是, 经丰富的研 究例证发现, 这一过程难免存在这样或那样的问题。与 此同时, 环境规制、生产效率提高、竞争力提升传递过 程明确指出, 但是不能综合分析过程中的成本分析, 仅 仅对遵守环境政策的相关成本进行了单一考虑，对于技 术进步等活动造成的额外投入漠不关心，更不上对于成 本抵偿效应有效性的科学论证。基于此, 有必要进一步 细化、拓展原有假说。

\subsection{3 环境规制的强绿色创新激励}

弱绿色创新激励的前提在于成本抵偿效应的存在， 其能够将波特假说的主要内容基本反映出来。站在假说 反对派的观念, 针对在一定时期内由于环境规制提高生 产效率而并非提高竞争力的问题可以进行集中批判, 如 果站在成本角度上, 新增成本超过节约成本的可能性比 较大，此时便难以成立波特假说。而总生产成本的增加， 对于企业原有利润水平的保持十分不利, 且很难调动企 业参与环保、减少环境污染的主观能动性。为此, 就需 要立足于原有环境规制, 确保政府干预、政策导向作用 的进一步增强 ${ }^{[5]}$ 。 


\section{4. 结束语}

现如今, 我国越来越重视环境问题, 在各方面的逐渐增 加了投入力度, 如政策实施、治污投资以及管理人员等, 从而促进了环境管理水平的显著提高, 为创新绿色技术 提供了源源不断的动力。但是, 在此过程中难免会存在 一系列问题, 如浪费、重复投入等, 由此也引发了各种 问题, 如不能合理的配置各资源要素、投入资源转换率 较低等, 最终造成在某个时期不利于最佳投入产出规模 的实现, 为此还需要进一步的提升与挖掘产出效益。综 上所述, 本文围绕绿色创新导向的环境规制, 提供有效 的理论与实证依据来制定更加有效的环境规制政策, 且 提供一定决策参考为相关部门促进针对性环境规制目 标的实现。

\section{REFERENCES}

[1] Xiaojun Ding, Yangting Fang. Research on the relationship between environmental regulation and green innovation in China--empirical analysis based on meta-analysis method[J]. Price Theory and Practice, 2018(06):34-37.

[2] Mingyu Liu, Baolong Yuan.The spatial heterogeneity effect of environmental regulation and green innovation efficiency-based on the data of industrial enterprises in yangtze river economic belt [J]. Journal of Finance and Accounting, 2018(24):144-153.

[3] Juanju Wang, Yu Zhang. Environmental regulation, willingness of green technology innovation and green technology innovation behavior [J]. Scientific Research, 2018,36(02):352-360.

[4] Zhengyue Hu, Wenjie Wu, Dongqin Li. Research on the impact of market incentive environmental regulation on enterprise green technology innovation-taking zhejiang province as an example [J]. Jiangsu Business Theory, 2017(11):110-114.

[5] Wenbin Peng, Jianglin Lu. Environmental regulation and green innovation policy: theoretical logic based on externality [J]. Social Sciences, 2017(10):73-83. 